\title{
NEER TOTAL SHOULDER REPLACEMENT IN RHEUMATOID ARTHRITIS
}

\author{
I. G. KELLY, R. S. FOSTER, W. D. FISHER
}

From the University Department of Orthopaedic Surgery, Glasgow

\begin{abstract}
Forty-two shoulders in 37 patients with polyarthritis were treated with Neer total shoulder replacements and reviewed 12 to 66 months afterwards. There was good pain relief and improvement in function, but the range of movement was less than that seen after replacements for osteoarthritis; this may have been related to the fact that $\mathbf{3 4}$ shoulders had abnormal rotator cuff tendons.

Although there was a high incidence of radiolucent lines around the glenoid component, there was no clinical evidence of loosening. There were a few complications, but on the whole we feel that the Neer total shoulder arthroplasty is a valuable procedure for a patient with polyarthritis.
\end{abstract}

Replacement arthroplasty of the shoulder is now a well established procedure. Most reports include several diagnostic categories, but Marmor (1977), Lettin (1981), Bodey and Yoeman (1983) and Pahle and Kvarnes (1985) have all discussed the use of shoulder arthroplasty in the rheumatoid patient. Problems with glenoid fixation have been encountered with a semi-constrained design (Lettin 1981), and most reports concern non-constrained arthroplasties. Because the function of a non-constrained arthroplasty (such as the Neer procedure) depends upon intact tendons and functioning muscles, it is important to analyse the operative results separately in polyarthritic patients where these tissues may be deficient.

\section{PATIENTS AND METHODS}

Between 1979 and 1984, 43 Neer total shoulder arthroplasties were performed on 38 patients with polyarthritis in the University Department of Orthopaedic Surgery, Glasgow; one patient died of unrelated causes within six months of surgery and has been excluded from the study. Thirty-five patients with $\mathbf{4 0}$ shoulder arthroplasties have been reviewed clinically and radiographically by one author (RSF) who had not been involved in the original surgery. The remaining two patients could not attend for

I. G. Kelly, MD, FRCS Ed, Senior Lecturer

University Department of Orthopaedic Surgery, Western Infirmary, Glasgow G11 6NT, Scotland.

R. S. Foster, MD

Oak Knoll Naval Hospital, San Francisco, California 94627, USA.

W. D. Fisher, MD, FRCS Ed, Associate Professor

Orthopaedic Surgery, McGill University, Montreal, Canada.

Requests for reprints should be sent to Mr I. G. Kelly.

(C) 1987 British Editorial Society of Bone and Joint Surgery $0301-620 \mathrm{X} / 87 / 5132 \$ 2.00$ review but both returned postal questionnaires and one also sent an up-to-date radiograph.

There were 30 women and seven men; their average age was 57 years (range 21 to 79 years). Follow-up ranged from 12 months to 66 months with a mean of 36 months. The diagnosis was classic seropositive rheumatoid arthritis in 41 cases and psoriatic polyarthropathy in the remaining case. The indication for surgery in all patients was severe pain in the shoulder associated with marked limitation of function. One patient had previously had a bursectomy, and all but two had involvement of other upper limb joints.

Patients were assessed pre-operatively, intra-operatively and postoperatively at two weeks, six months, one year and then annually, using Neer's system as modified by Cofield (Neer, Watson and Stanton 1982) together with anteroposterior and axillary radiographs of the shoulder. The pre-operative radiographs were assessed using the classification described by Larsen, Dale and Eek (1977): 14 shoulders showed deep erosion to the subchondral plate with the joint space reduced to a line (Grade IV), while 28 had total disorganisation of the joint with major deterioration or resorption of bone (Grade V).

Operative technique. Most of the arthroplasties were carried out in operating theatres with laminar flow ventilation and all patients received 48 hours of prophylactic antibiotics.

The operative technique used differs little from that described by Cofield (1984), although it was usually necessary to extend the incision in the subscapularis tendon into the supraspinatus portion of the cuff to gain adequate access to the joint. The coraco-acromial ligament was left intact in 13 arthroplasties, and is now divided only when a tear of the cuff needs to be repaired. The glenoid components were cemented into position 
using injected Surgical Simplex (Howmedica) in 28 cases and manually inserted $\mathrm{CMW}$ in 14 . A secure press-fit could not be obtained in only one of the humeral components, which was then cemented.

At the end of the operation a broad arm sling and a body swathe are applied. Passive mobilisation out of the sling is begun on the fourth postoperative day. After discharge from hospital, the patient continues regular exercises at home, supplemented with intermittent outpatient physiotherapy in hospital for between three and six months.

\section{RESULTS}

A summary of the operative findings is given in Table $I$. The bone was usually osteoporotic, and deficiency of glenoid bone stock was noted in 26 shoulders $(62 \%)$; of these, two required bone grafting. Rotator cuff disease (due to rheumatoid arthritis) was present in 34 shoulders $(81 \%)$, although only eight $(19 \%)$ had cuff tears. The clinical and functional findings are summarised in Tables II and III.

Table I. Findings during 42 arthroplasties in 37 patients

\begin{tabular}{lr} 
& Number \\
\hline Rotator cuff & \\
$\quad$ Normal & 8 \\
Thin & 26 \\
Minor tear & 5 \\
Major tear & 3 \\
Biceps tendon & \\
$\quad$ Normal & 2 \\
Frayed & 6 \\
Torn & 34 \\
Glenoid bone & \\
$\quad$ Normal & 16 \\
Deficient & 26 \\
\hline
\end{tabular}

Pain relief was very good, 36 shoulders $(88 \%)$ having no pain or pain with unusual activities only. Five patients still had moderate pain, four having clinical evidence of a non-functional rotator cuff and one having no identifiable cause of pain. One patient developed severe pain in the early postoperative period secondary to failure of the rotator cuff, and later required revision to an arthrodesis.

For the entire group, gains in the range of active movement were modest for flexion but large for both medial (internal) and lateral (external) rotation and extension (Table II). Five patients lost more than $10^{\circ}$ of flexion, and in six patients the flexion range was unchanged. All patients improved their ranges of medial and lateral rotation by at least $10^{\circ}$.

Function was assessed according to the patient's ability to perform activities of daily living (Table III). Each of the nine activities was scored four points if normal, three if achieved with some difficulty, two if an aid was needed, and one point if the patient was unable to perform the task; a perfect score would be 36 points.
Table II. Pre-operative and postoperative ranges of movement

\begin{tabular}{|c|c|c|c|c|c|}
\hline & \multicolumn{5}{|c|}{ Shoulder movement (degrees) } \\
\hline & \multicolumn{2}{|c|}{ Pre-operative } & \multicolumn{2}{|c|}{ Postoperative } & \multirow{2}{*}{$\begin{array}{l}\text { Gain } \\
\text { (average) }\end{array}$} \\
\hline & Average & Range & Average & Range & \\
\hline Flexion & 55 & $0-110$ & 75 & 25170 & 20 \\
\hline Abduction & - & - & 68 & $30-115$ & - \\
\hline Lateral rotation & 11 & $-30-50$ & 40 & $5-80$ & 29 \\
\hline Medial rotation & - & GT-T12 & - & B-T6 & - \\
\hline Extension & 18 & $0-40$ & 50 & $15-80$ & 32 \\
\hline
\end{tabular}

GT, greater trochanter; B, buttock

Note: abduction was not measured pre-operatively; range refers to movement seen in all patients

Before operation the mean score and standard deviation was $16 \pm 3$ (range 11-23); after operation these values were $30 \pm 6$ (range 14-40). No patient's function deteriorated after operation despite the fact that function was frequently affected by disease in the other upper limb joints.

The finding of a rotator cuff tear at operation in 13 shoulders resulted in a smaller postoperative range of flexion than that seen in the remainder of the group (a mean of $64^{\circ}$ compared to $77^{\circ}$ ), but was associated with similar levels of function (28 points compared with 31 points). Seven patients, only one of whom had a cuff tear at operation, were found to have a non-functional cuff postoperatively: their mean flexion range was $28^{\circ}$ (compared with $87^{\circ}$ for the rest of the shoulders) and their mean functional score was 25 points. All seven could wash the opposite axilla, four could attend to their perineal toilet, six could use an eating utensil, but only one could comb her hair. None could use the hand above shoulder level.

When asked for their opinions of the procedure, all but one of the patients felt that they were "much improved", and no patient regretted having undergone the operation.

Table III. Assessment of functional ability in 41 shoulders

\begin{tabular}{lll}
\hline & \multicolumn{2}{l}{ Number of shoulders } \\
\cline { 2 - 3 } Activity & Pre-operative & Postoperative \\
\hline Use back pocket & 10 & 37 \\
Attend to perineal toilet & 17 & 34 \\
Wash opposite axilla & 12 & 39 \\
Use eating utensil & 16 & 38 \\
Comb hair & 5 & $22^{*}$ \\
Carry $4.5-7.0$ kg at side & 9 & 24 \\
Use hand at shoulder level & 5 & 29 \\
Use hand over head & 3 & 12 \\
Lie on affected side & 10 & 30 \\
\hline
\end{tabular}

- With the use of a long-handled comb, another eight patients could comb their hair 


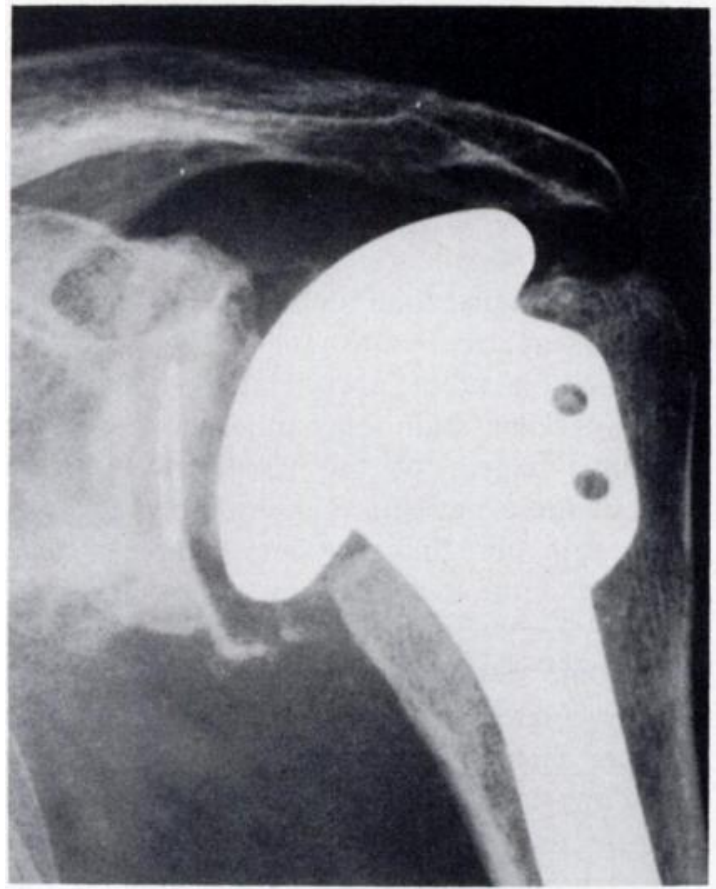

Fig. 1

A Neer total shoulder arthroplasty showing typical articulation.

Radiographic analysis. According to Neer et al. (1982), it appears that the prosthetic humeral head should be centred with the junction of its middle and lower thirds opposite the centre of the wire marker on the glenoid component (Fig. 1). The amount of superior subluxation was measured: pre-operatively, all shoulders showed superior subluxation; postoperatively, 24 arthroplasties showed no evidence of subluxation, but in some it was apparent on the first radiograph, while in others it appeared to develop it as a result of the tilt of the glenoid component. Four of the five shoulders with subluxation of more than $5 \mathrm{~mm}$ had evidence of a non-functioning rotator cuff; in the fifth the humeral component had sunk into the humeral shaft.

Radiolucent lines were found around the glenoid component in 33 patients (Table IV). Eleven joints with a lucent line at the final review had had some evidence of lucency on the radiographs at two weeks after operation, 21 showed no progression in the extent or thickness of the lucency and four showed progression which ceased within the first year. Six patients, all with less than 24 months' follow-up, still showed progression at the time of review. However, only one of these had a lucency which completely surrounded the component.

Of the patients with uncemented humeral components, 21 had no radiolucency and 10 had a complete lucency; no lucency was progressive or wider than $1.5 \mathrm{~mm}$.

Complications. These included two intra-operative fractures, one of the glenoid and one of the humerus.

Non-functional rotator cuffs after operation were diagnosed clinically in seven shoulders, and in two of
Table IV. Radiolucent zones seen in radiographs of patients

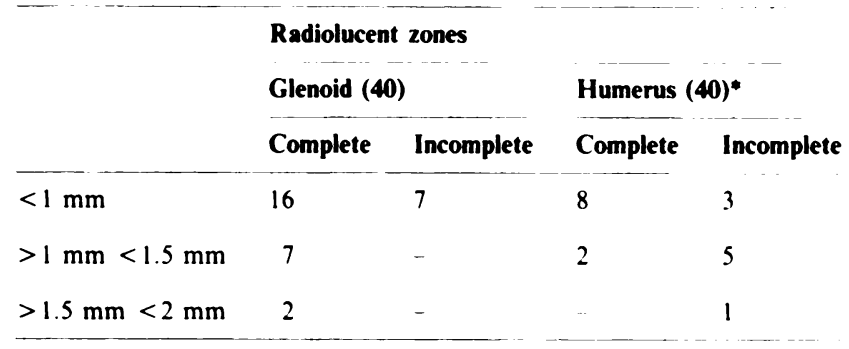

- Radiographs for the one patient treated with a cemented humeral component were not available

these arthrography confirmed a ruptured cuff (preoperatively, the cuff had been intact in one, thin in five and the site of a major tear in one).

One patient with severe postoperative pain required re-operation; this was a 65 -year-old woman with active seropositive rheumatoid arthritis. At the initial operation the rotator cuff was noted to be very thin and its repair was tight. Six weeks after operation she experienced sudden pain in the shoulder with loss of active movements due to failure of the rotator cuff. Six months after a failed attempt at rotator cuff repair, the prosthesis was removed for pain. Unfortunately, within another six months the pseudarthrosis became painful because of impingement and fusion was performed with a successful outcome.

\section{DISCUSSION}

The Neer total shoulder replacement gave relief from pain and functional improvement in the majority of our patients with polyarthritis. However, using the criteria of Neer et al. (1982), only six patients in our series would have achieved even a "satisfactory" grading and, using the less stringent "limited goals" criteria of Cofield (1984), only 20 shoulders would have been graded successful. The lack of recovery of elevation (flexion or abduction) is the main reason for the poor grading of our patients.

Movements at the glenohumeral joint are dependent upon muscle power and the coupling of muscular action (Inman, Saunders and Abbott 1944; Walker 1982). Because of their disease, rheumatoid patients lack muscle power and it is to be expected that recovery of active movement, especially against gravity, will be poor. Our findings are similar to those of other authors who reported limited elevation with moderate gain in medial and lateral rotation (Marmor 1977; Lettin 1981 ; Warren, Ranawat and Inglis 1982; Bodey and Yoeman 1983; Cofield 1984; Pahle and Kvarnes 1985). This gain explains the good functional recovery since very few activities of daily living require the hand to be moved above the top of the head, but most require rotation at the shoulder.

We feel that the grading of results proposed by both Neer et al. (1982) and Cofield (1984), which depends 
upon the improved movement in patients with noninflammatory arthropathies, is inappropriate for patients with rheumatoid arthritis. Our results show poor correlation between the range of movement and the functional recovery, as demonstrated in other studies of rheumatoid arthritis (Badley, Wagstaff and Wood 1984).

However, a certain minimum range of movement is necessary for function. Without the rotator cuff the deltoid muscle has only limited ability to elevate the arm against gravity (Bechtol 1980). The high incidence of cuff abnormalities in our patients is similar to that reported by Cofield (1984) and must contribute to the limitation of movement. A small prosthetic humeral head was often used to permit the repair of a thin rotator cuff, and may have resulted in too small a lever arm to allow the deltoid and the weakened cuff to function effectively. Postoperative failure of the cuff may reflect either the poor quality of the cuff or our management of its closure. However, neither the state of the cuff at operation nor the recorded quality of the cuff closure allowed prediction of the failure.

Superior subluxation of the humeral head is usually regarded as a sign of rotator cuff disease, and Pahle and Kvarnes (1985) referred to it as being indicative of a "technical failure" when seen after shoulder arthroplasty. Rotator cuff rupture or altered balance between the deltoid and the cuff muscles directs the resultant force at the glenohumeral joint more superiorly, and encourages superior movement of the humeral head relative to the glenoid. Retention of the coraco-acromial ligament might be expected to limit this. Four of the 17 patients with subluxation had evidence of a nonfunctional cuff; only one had not had the ligament divided and all showed progressive subluxation. Twelve others had non-progressive subluxation as a result of the orientation of the glenoid component. Superior subluxation from whatever cause predisposes to impingement of the greater tuberosity on the acromion during elevation, thus reducing the range of movement available at the glenohumeral joint whether the cuff is functional or not.

Despite the factors limiting glenohumeral movements, the absence of pain made more scapulothoracic movement available to all our patients, allowing even those with non-functional rotator cuffs to regain a useful level of function.

In common with Cofield (1984) we found radiolucent lines around the glenoid components in $80 \%$ of patients (Table IV). A high proportion were present as early as two weeks after operation, suggesting that our cementing technique may have been inadequate. However, no difference was seen in the incidence of lucent lines between those cemented using injection techniques and those in whom it was inserted manually. Amstutz, Sew Hoy and Clarke (1981) reported a similarly high incidence of radiolucent lines despite using a meticulous cementing technique. Recent studies of the polyethylene components of knee arthroplasties have suggested that it is only the progressive lucent line, irrespective of its width, that is related to loosening (Ryd 1986). We are encouraged by the lack of clinical loosening and the small proportion of cases showing progressive radiolucent lines, although doubts must remain about the durability of the glenoid component.

We conclude that total replacement of the shoulder in rheumatoid arthritis, using a non-constrained prosthesis, is a valuable procedure yielding good functional results and excellent pain relief in joints with advanced disease. Involvement of the rotator cuff is almost universal in these patients and probably explains the failure to regain more than a moderate range of elevation but, even in the presence of an impaired cuff, gratifying results have been obtained. Complications have been few, and could perhaps be further reduced by closer attention to rotator cuff reconstruction. Only time will tell whether glenoid component loosening will be a significant problem, but salvage by arthrodesis is possible in the event of failure. Non-constrained shoulder arthroplasty now seems to merit a place alongside other joint replacements in the surgical management of rheumatoid arthritis.

\section{REFERENCES}

Amstutz HC, Sew Hoy AL, Clarke IC. UCLA anatomic total shoulder arthroplasty. Clin Orthop 1981;155:7-20.

Badley EM, Wagstaff S, Wood PH. Measures of functional ability (disability) in arthritis in relation to impairment of range of joint movement. Ann Rheum Dis 1984;43:563-9.

Bechtol CO. Biomechanics of the shoulder. Clin Orthop $1980 ; 146: 37-41$.

Bodey WN, Yoeman PM. Prosthetic arthroplasty of the shoulder. Acta Orthop Scand 1983:54:900-3.

Cofield RH. Total shoulder arthroplasty with the Neer prosthesis. $J$ Bone Joint Surg [Am] 1984:66-A :899-906.

Inman VT, Saunders JB deCM, Abbott LC. Observations on the function of the shoulder joint. J Bone Joint Surg 1944;28:1-30.

Larsen A, Dale K, Eek M. Radiographic evaluation of rheumatoid arthritis and related conditions by standard reference films. Acto Radiol [Diagn] (Stockh) 1977;18:481-91

Lettin A. Shoulder replacement in rheumatoid arthritis. Reconstr Surg Traumatol $1981 ; 18: 55-62$.

Marmor L. Hemiarthroplasty for the rheumatoid shoulder joint. Clin Orthop 1977;122:201-3.

Neer CS II, Watson KC, Stanton FJ. Recent experience in total shoulder replacement. J Bone Joint Surg [Am] 1982;64-A :319-37.

Pahle JA, Kvarnes L. Shoulder replacement arthroplasty. Ann Chir Gynaecol 1985:198 Suppl 74:85-9.

Ryd L. Micromotion in knee arthroplasty: a roentgen stereophotogrammetric analysis of tibial component fixation. Acta Orthop Scand 1986:57 Suppl 220.

Walker PS. Some bioengineering considerations of prosthetic replacement for the glenohumeral joint. In: Inglis AE, ed. Symposium on the total joint replacement of the upper extremity New York, 1979. St Louis etc. CV Mosby, 1982:25-32.

Warren RF, Ranawat CS, Inglis AE. Total shoulder replacement indications and results of the Neer nonconstrained prosthesis. In : Inglis AE, ed. Total joint replacement of the upper extremity. St Louis etc: CV Mosby, 1982:56-67. 